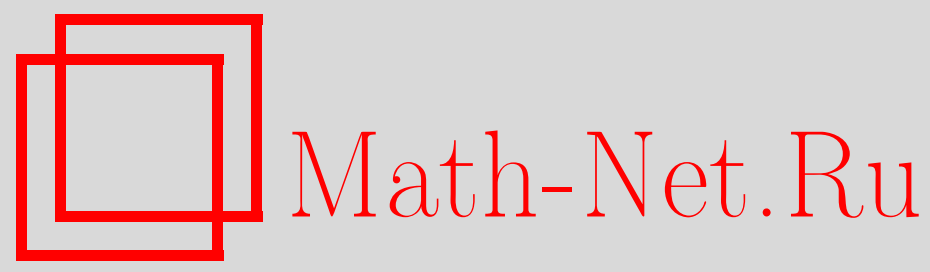

М. Б. Хрипунова, Об одной мультипликативной функции на множестве сдвинутых простых чисел, Матем. заметки, 1998, том 64, выпуск 3, 457-464

DOI: https://doi.org/10.4213/mzm1417

Использование Общероссийского математического портала Math-Net.Ru подразумевает, что вы прочитали и согласны с пользовательским соглашением http://www.mathnet.ru/rus/agreement

Параметры загрузки:

IP : 44.207 .124 .84

26 апреля 2023 г., $17: 05: 05$

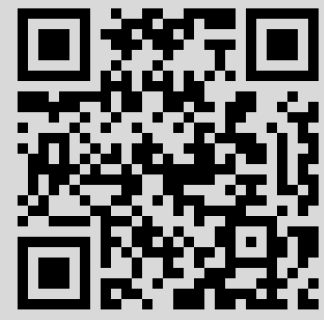




\section{ОБ ОДНОЙ МУЛЬТИПЛИКАТИВНОЙ ФУНКЦИИ НА МНОЖЕСТВЕ СДВИНУТЫХ ПРОСТЫХ ЧИСЕЛ}

\section{М.Б. Хрипунова}

Доказано, что если $f(n)$ - мультипликативная функция, принимающая на множестве простых чисел значение $\xi$, где $\xi^{3}=1, \xi \neq 1$ и $f^{3}\left(p^{r}\right)=1$ при $r \geqslant 2$, то существует $\theta \in(0,1)$, для которого

$$
\left|\sum_{p \leqslant x} f(p+1)\right| \leqslant \theta \pi(x), \quad \text { где } \pi(x)=\sum_{p \leqslant x} 1 .
$$

Библиография: 6 названий.

Существует гипотеза о том, что если $f(n)$-мультипликативная функция и $f(p+1)=1$ для любого простого $p$, то $f(n) \equiv 1$. Мейеру [1] удалось доказать, что в этом случае существует $k \leqslant 4$, при котором $f^{k}(n) \equiv 1$.

Можно рассмотреть другой вариант этой гипотезы. Предположим, что $|f(n)| \leqslant 1$ и

$$
\lim _{x \rightarrow \infty} \frac{1}{\pi(x)} \sum_{p \leqslant x} f(p+1)=1, \quad \text { где } \pi(x)=\sum_{p \leqslant x} 1 .
$$

Тогда ожидается, что $f(n) \equiv 1$. В этом случае Хильдебрант [2] показал, что существует $k \leqslant c_{1}$, где $c_{1}$ - некоторая большая постоянная, при котором $f^{k}(n) \equiv 1$. Н. М. Тимофеев [3] доказал, что этот результат верен при $k \leqslant 4$. Дальнейшие исследования в этом направлении и интересные следствия можно найти в работе Эллиотта [4]. Тем не менее в настоящее время вопрос о верности неравенства

$$
\lim _{x \rightarrow \infty} \sup \frac{1}{\pi(x)}\left|\sum_{p \leqslant x} f(p+1)\right|<1, \quad \text { где } f(n) \not \equiv 1, \quad(n, 2)=1,
$$

остается открытым. Так неравенство (1) не доказано, в частности, для функций $f(n)$ таких, что $f^{3}(n) \equiv 1$ или $f^{2}(n) \equiv 1$. Например, неравенство (1) не доказано для $\lambda(n)-$ функции Лиувилля $\lambda\left(p^{r}\right)=(-1)^{r}$; т.е. пока не удалось получить нетривиальную оценку для суммы

$$
\sum_{p \leqslant x} f(p+1)
$$

если $f$ на простых числах принимает значение -1 .

Целью настоящей работы является следующий результат.

Работа выполнена при финансовой поддержке Российского фонда фундаментальных исследований, грант № 96-01-00502. 
Теорема. Если $f(n)$-мультипликативная функиия, принимающая на простых числах значение $\zeta$, где $\zeta^{3}=1, \zeta \neq 1 u f^{3}\left(p^{r}\right)=1$ при $r \geqslant 2$, то существует постоянная $0<\theta<1$ такая, что при $x \geqslant x_{0}$

$$
\left|\sum_{p \leqslant x} f(p+1)\right| \leqslant \theta \pi(x) .
$$

ЗАмЕчАниЕ. Если $\zeta^{k}=1, \zeta$ - первообразный корень $k$-й степени из единицы, $k>3$, то этот результат вытекает из работ Тимофеева [3] и Эллиотта [4].

ДокАЗАТЕЛЬСТво. Будем использовать решето и метод Чена, с помошью которого он получил оценку снизу для чисел $p+2, p \leqslant x$, имеюших не более двух простых делителей. Мы будем применять его, следуя схеме работы [5].

Итак, пусть $P_{2}$ - числа, имеюшие не более двух простых делителей, каждый из которых больше $x^{\beta}, \beta=1 / 10, n_{1}$ - четные числа, $n_{1} \leqslant x^{\alpha}, \alpha \leqslant \beta$, удовлетворяюшие некоторым условиям, которые введем позже.

Обозначим

$$
P(y)=\prod_{p \leqslant y} p, \quad S\left(n_{1}\right)=\left|\left\{p: p \leqslant x, p+1=n_{1} P_{2}\right\}\right| .
$$

Докажем неравенство

$$
\sum_{n_{1}}^{\prime} S\left(n_{1}\right) \geqslant \frac{\pi(x) \Lambda}{\ln x}(0.64+O(\alpha)) \sum_{n_{1}}^{\prime} \frac{g\left(n_{1}\right)}{\varphi\left(n_{1}\right)},
$$

где

$$
g\left(n_{1}\right)=\prod_{\substack{p \mid n_{1} \\ p \neq 2}}\left(1-\frac{1}{p-1}\right)^{-1}\left(1-\frac{1}{p}\right), \quad g\left(2^{r}\right)=1, \quad \Lambda=\prod_{p>2}\left(1-\frac{1}{(p-1)^{2}}\right) .
$$

Здесь и ниже $\sum^{\prime}$ обозначает, что $n_{1}$ пробегает некоторое подмножество, которое будет выбрано позже, и $n_{1} \leqslant x^{\alpha}, n_{1}$ - четное, $\alpha \leqslant 0.1$ - абсолютная постоянная, входящая в символ $O(\alpha)$.

Имеем

$$
\begin{aligned}
& S\left(n_{1}\right) \geqslant\left|\left\{p: p \leqslant x, p+1 \equiv 0\left(\bmod n_{1}\right),\left(\frac{p+1}{n_{1}}, P\left(x^{\beta}\right)\right)=1\right\}\right| \\
& -\frac{1}{2} \sum_{\left(x / n_{1}\right)^{1 / 10} \leqslant p_{1} \leqslant\left(x / n_{1}\right)^{1 / 3}}\left|\left\{p: p \leqslant x, p+1 \equiv 0\left(\bmod p_{1} n_{1}\right),\left(\frac{p+1}{p_{1} n_{1}}, P\left(x^{\beta}\right)\right)=1\right\}\right| \\
& -\frac{1}{2} \mid\left\{p: p \leqslant x, p+1=n_{1} p_{1} p_{2} p_{3},\left(\frac{x}{n_{1}}\right)^{1 / 10} \leqslant p_{1} \leqslant\left(\frac{x}{n_{1}}\right)^{1 / 3}\right. \\
& \left.\left(\frac{x}{n_{1}}\right)^{1 / 3} \leqslant p_{2} \leqslant\left(\frac{x}{p_{1} n_{1}}\right)^{1 / 2}\right\} \mid+O\left(\left(\frac{x}{n_{1}}\right)^{1-\beta}\right) .
\end{aligned}
$$

Это неравенство подобно неравенству (2.1) из [6, с. 321] и проверяется аналогично. 
Обозначим первое слагаемое в правой части неравенства (3) через $S_{1}\left(n_{1}\right)$ и, пользуясь линейным решетом, найдем для него оценку снизу (см. [6, теорема 8.4]). Возьмем

$$
\xi^{2}=\frac{\sqrt{x}}{n_{1} \log ^{A} x}, \quad \frac{\rho(d)}{d}=\frac{\varphi\left(n_{1}\right)}{\varphi\left(n_{1} d\right)}
$$

$\varphi(n)$ - функция Эйлера, $X=\pi(x) / \varphi(n), z=x^{\beta} / n_{1}$. Тогда имеем

$$
\begin{aligned}
\sum_{n_{1}}^{\prime} S_{1}\left(n_{1}\right) \geqslant & \sum_{n_{1}}^{\prime} \pi(x) \frac{1}{\varphi(x)} \prod_{p<x^{\beta}}\left(1-\frac{\varphi\left(n_{1}\right)}{\varphi\left(n_{1} p\right)}\right)\left(f\left(\frac{\log \xi^{2}}{\log z}\right)-B \frac{L}{(\log \xi)^{1 / 14}}\right) \\
& -\sum_{n_{1}}^{\prime} \sum_{\substack{d \leqslant \xi^{2} \\
d \mid P\left(x^{\beta}\right)}} 3^{\omega(d)}\left|\sum_{\substack{p \leqslant x \\
p \equiv-1\left(\bmod d n_{1}\right)}} 1-\frac{\varphi\left(n_{1}\right)}{\varphi\left(n_{1} d\right)} \frac{\pi(x)}{\varphi\left(n_{1}\right)}\right| .
\end{aligned}
$$

По теореме Виноградова-Бомбьери [5] получим, что остаток есть

$$
O\left(\frac{x}{\log ^{B} x}\right)
$$

где $B=B(A)-$ положительная постоянная. Поэтому

$$
\begin{aligned}
\sum_{n_{1}}^{\prime} S\left(n_{1}\right) \geqslant & \frac{1}{2} \prod_{2<p<x^{\beta}}\left(1-\frac{1}{1-p}\right) \pi(x) \sum_{n_{1}}^{\prime} \frac{1}{\varphi\left(n_{1}\right)} \prod_{\substack{p \mid n_{1} \\
p \neq 2}}\left(1-\frac{1}{p}\right)\left(1-\frac{1}{p-1}\right)^{-1} \\
& \times\left(f\left(\frac{\log \xi^{2}}{\log x^{\beta}}\right)-B \frac{L}{(\log x)^{1 / 14}}\right)+O\left(\frac{x}{\log ^{B} x}\right)
\end{aligned}
$$

Далее проверим вьполнение условий линейного решета (см. [6, с. 228]). Имеем

$$
\sum_{\omega \leqslant p<z} \frac{\varphi\left(n_{1} p\right)}{\varphi(p)} \log p=\sum_{\substack{\omega \leqslant p<z \\ p \mid n_{1}}} \frac{\log p}{p}+\sum_{\substack{\omega \leqslant p<z \\ p \nmid n_{1}}} \frac{\log p}{p-1}=\log \frac{z}{\omega}+O(1)
$$

т.е. условие выполнено с $L=O(1)$.

Так как $f(t)$ - монотонно возрастающая и дифференцируемая функция, имеем

$$
f\left(\frac{\log \xi^{2}}{\log x^{\beta}}\right) \geqslant f\left(\frac{1 / 2-\alpha}{\beta}\right)+O\left(\frac{\log _{2} x}{\log x}\right) \geqslant f(5)+O(\alpha) .
$$

Здесь мы воспользовались тем, что $\beta=0.1$. Окончательно для первой суммы получим

$$
\sum_{n_{1}}^{\prime} S_{1}\left(n_{1}\right) \geqslant \frac{1}{2} \pi(x)(f(5)+O(\alpha)) \prod_{2<p<z}\left(1-\frac{1}{p-1}\right) \sum_{n_{1}}^{\prime} \frac{g\left(n_{1}\right)}{\varphi\left(n_{1}\right)}+O\left(\frac{x}{\log ^{\beta} x}\right) .
$$


Оценим сверху $S_{1}^{\prime}\left(p_{1} n_{1}\right)$ - вторую сумму в правой части неравенства $(3)$, просуммировав ее по $n_{1}$. Применив оценку сверху линейного решета (см. [6, теорема 8.4$]$ ), получим

$$
\begin{aligned}
& \sum_{n_{1}}^{\prime} \frac{1}{2} \sum_{\left(x / n_{1}\right)^{1 / 10} \leqslant p_{1} \leqslant\left(x / n_{1}\right)^{1 / 3}} S_{1}^{\prime}\left(p_{1} n_{1}\right) \\
& \leqslant \frac{1}{2} \sum_{n_{1}}^{\prime} \sum_{\left(x / n_{1}\right)^{1 / 10} \leqslant p_{1} \leqslant\left(x / n_{1}\right)^{1 / 3}} \frac{\pi(x)}{\varphi\left(p_{1} n_{1}\right)} \prod_{p<z}\left(1-\frac{\varphi\left(n_{1}\right)}{\varphi\left(n_{1} p\right)}\right) \\
& \times\left(F\left(\frac{\log \xi^{2}}{\log x^{\beta}}\right)+O\left(\frac{L}{(\log \xi)^{1 / 14}}\right)\right) \\
& +\sum_{n_{1}}^{\prime} \sum_{\left(x / n_{1}\right)^{1 / 10} \leqslant p_{1} \leqslant\left(x / n_{1}\right)^{1 / 3}} \sum_{d \leqslant \xi^{2}} 3^{\omega(d)}\left|\sum_{\substack{p \leqslant x \\
p \equiv 1\left(\bmod n_{1} p_{1} d\right)}} 1-\frac{\varphi\left(n_{1} p_{1}\right)}{\varphi\left(n_{1} p_{1} d\right)} \frac{\pi(x)}{\varphi\left(n_{1} p_{1}\right)}\right| .
\end{aligned}
$$

Мы применили линейное решето с

$$
\frac{\rho(d)}{d}=\frac{\varphi\left(n_{1} p_{1}\right)}{\varphi\left(n_{1} p_{1} d\right)}, \quad X=\frac{\pi(x)}{\varphi\left(n_{1} p_{1}\right)}, \quad L=O(1), \quad \xi^{2}=\frac{\sqrt{x}}{n_{1} p_{1} \log ^{A} x}
$$

Оценим остаток, как и в первом случае. Он будет равен $O\left(x \log ^{-B} x\right) . F(t)$-монотонно убывающая и дифференцируемая функция, поэтому

$$
F\left(\frac{\log \xi^{2}}{\log z}\right) \leqslant F\left(5-10 \frac{\log p_{1}}{\log x}\right)+O(\alpha) .
$$

Следовательно, исследуемая сумма

$$
\begin{aligned}
& \leqslant \frac{\pi(x)}{4} \prod_{2<p<z}\left(1-\frac{1}{p-1}\right) \sum_{n_{1}}^{\prime} \frac{1}{\varphi\left(n_{1}\right)} \prod_{\substack{p \mid n_{1} \\
p \neq 2}}\left(1-\frac{1}{p}\right)\left(1-\frac{1}{p-1}\right)^{-1} \\
& \quad \times \sum_{\left(x / n_{1}\right)^{1 / 10} \leqslant p_{1} \leqslant\left(x / n_{1}\right)^{1 / 3}} \frac{1}{\varphi\left(p_{1}\right)}\left(F\left(5-10 \frac{\log p_{1}}{\log x}\right)+O(\alpha)\right)+O\left(\frac{x}{\log ^{B} x}\right) .
\end{aligned}
$$

Преобразуем сумму по $p_{1}$. Учитьвая, что части суммы по $p_{1} \in\left[\left(x / n_{1}\right)^{1 / 3}, x^{1 / 3}\right]$ и $p_{1} \in\left[\left(x / n_{1}\right)^{1 / 10}, x^{1 / 10}\right]$ есть $O(\alpha)$, применив суммирование по Абелю, получим, что эта сумма равна

$$
\int_{3}^{10} F\left(5-\frac{10}{t}\right) \frac{d t}{t}+O(\alpha)
$$

Итак, используя (4), имеем

$$
\begin{gathered}
\sum_{n_{1}}^{\prime} S_{1}\left(n_{1}\right)-\frac{1}{2} \sum_{n_{1}}^{\prime} \sum_{\left(x / n_{1}\right)^{1 / 10} \leqslant p \leqslant\left(x / n_{1}\right)^{1 / 3}} S^{\prime}\left(n_{1} p\right) \geqslant \frac{\pi(x)}{2} \prod_{2<p<z}\left(1-\frac{1}{p-1}\right) \\
\times \sum_{n_{1}}^{\prime} \frac{g\left(n_{1}\right)}{\varphi\left(n_{1}\right)}\left(f(5)-\frac{1}{2} \int_{3}^{10} F\left(5-\frac{10}{t}\right) \frac{d t}{t}+O(\alpha)\right)+O\left(\frac{x}{\log ^{B} x}\right) .
\end{gathered}
$$


Исследуем третье слагаемое в $(3)$, обозначив его через $S_{0}\left(n_{1}\right)$. Имеем

$$
\begin{aligned}
S_{0}\left(n_{1}\right) \leqslant & \frac{1}{2} \mid\left\{p: p_{1} p_{2} p_{3} \leqslant \frac{x+1}{n_{1}},\left(\frac{x}{n_{1}}\right)^{1 / 10} \leqslant p_{1} \leqslant\left(\frac{x}{n_{1}}\right)^{1 / 3},\right. \\
& \left.\left(\frac{x}{n_{1}}\right)^{1 / 3} \leqslant p_{2} \leqslant\left(\frac{x}{n_{1}}\right)^{1 / 2},\left(n_{1} p_{1} p_{2} p_{3}-1, P(\sqrt{x})\right)=1\right\} \mid+\sqrt{x} .
\end{aligned}
$$

Для его оценки применим опять линейное решето, взяв

$$
\begin{gathered}
X=\left|\left\{p_{1} p_{2} p_{3} \leqslant \frac{x+1}{n_{1}},\left(\frac{x}{n_{1}}\right)^{1 / 10} \leqslant p_{1} \leqslant\left(\frac{x}{n_{1}}\right)^{1 / 3},\left(\frac{x}{n_{1}}\right)^{1 / 3} \leqslant p_{2} \leqslant\left(\frac{x}{n_{1}}\right)^{1 / 2}\right\}\right|, \\
\frac{\rho(p)}{p}=\left\{\begin{array}{ll}
\frac{1}{\varphi(p)}, & \text { если } p \nmid n_{1}, \\
0, & \text { в противном случае },
\end{array} \quad \xi^{2}=\frac{\sqrt{x}}{n_{1} \log ^{A} x}, \quad z=\xi .\right.
\end{gathered}
$$

Получим

$$
\begin{aligned}
\frac{1}{2} \sum_{n_{1}}^{\prime} S_{0}\left(n_{1}\right) \leqslant & \frac{1}{2} \sum_{n_{1}}^{\prime} X \prod_{\substack{p \leqslant \xi \\
p \nmid n_{1}}}\left(1-\frac{1}{p-1}\right)\left(F(2)+O\left(\frac{1}{(\log x)^{1 / 14}}\right)\right) \\
& +\sum_{n_{1}}^{\prime} \sum_{\substack{d \leqslant \xi^{2} \\
d \mid P(z) \\
\left(d, n_{1}\right)=1}} 3^{\omega(d)}\left|X(d)-\frac{1}{\varphi(d)} X\right|,
\end{aligned}
$$

где через $X(d)$ обозначено число чисел из $X$, удовлетворяющих условию $n_{1} p_{1} p_{2} p_{3} \equiv$ $1(\bmod d)$.

После применения леммы 3 из [5] получим, что вторая сумма в последнем выражении равна $O\left(x \log ^{-B} x\right)$.

Так как

$$
\begin{aligned}
X= & \frac{1}{n_{1}} \sum_{\left(x / n_{1}\right)^{1 / 10} \leqslant p_{1} \leqslant\left(x / n_{1}\right)^{1 / 3}} \sum_{\left(x / n_{1}\right)^{1 / 3} \leqslant p_{2} \leqslant\left(x / n_{1}\right)^{1 / 2}}\left(\frac{x}{p_{1} p_{2} \log \left(x /\left(n_{1} p_{1} p_{2}\right)\right)}\right) \\
& \left.+O\left(\frac{x}{p_{1} p_{2} \log ^{2} x}\right)\right),
\end{aligned}
$$

используя лемму 11.1 из [6], в силу которой

$$
\sum_{\left(x / n_{1}\right)^{1 / 10} \leqslant p_{1} \leqslant\left(x / n_{1}\right)^{1 / 3}} \sum_{\left(x / n_{1}\right)^{1 / 3} \leqslant p_{2} \leqslant\left(x / n_{1}\right)^{1 / 2}} \frac{1}{p_{1} p_{2} \log \left(x /\left(n_{1} p_{1} p_{2}\right)\right)} \leqslant \frac{0.493}{\log \left(x / n_{1}\right)},
$$

получим

$$
\frac{1}{2} \sum_{n_{1}}^{\prime} S_{0}\left(n_{1}\right) \leqslant \frac{1}{2} \frac{0.493}{\log x} \prod_{2<p<x^{1 / 4}}\left(1-\frac{1}{p-1}\right) \sum_{n_{1}}^{\prime} \frac{g\left(n_{1}\right)}{\varphi\left(n_{1}\right)}(F(2)+O(\alpha))+O\left(\frac{x}{\log ^{B} x}\right) .
$$


А теперь, учитывая, что по формуле Мертенса

$$
\prod_{p \leqslant x}\left(1-\frac{1}{p}\right)=\frac{e^{-\gamma}}{\ln x}\left(1+O\left(\frac{1}{\ln x}\right)\right)
$$

где $\gamma$ - постоянная Эйлера, из (3) и (5) находим

$$
\begin{aligned}
\sum_{n_{1}}^{\prime} S\left(n_{1}\right) \geqslant & 2 \Lambda \frac{x}{\log ^{2} x} \sum_{n_{1}}^{\prime} \frac{1}{\varphi\left(n_{1}\right)} g\left(n_{1}\right)\left(10 e^{-\gamma}\left(f(5)-\frac{1}{2} \int_{3}^{10} F\left(5-\frac{10}{t}\right) \frac{d t}{t}\right)\right. \\
& \left.-\frac{1}{2} \cdot 0.493 \cdot 4 \cdot e^{-\gamma} F(2)+O(\alpha)\right)+O\left(\frac{x}{\log ^{B} x}\right) .
\end{aligned}
$$

Здесь мы воспользовались тем, что

$$
\prod_{2<p \leqslant x}\left(1-\frac{1}{p-1}\right)=2\left(\Lambda+O\left(\frac{1}{x}\right)\right) \prod_{p \leqslant x}\left(1-\frac{1}{p}\right) .
$$

Так как (см. [6, с. 323])

$$
20 e^{-\gamma}\left(f(5)-\frac{1}{2} \int_{3}^{10} F\left(5-\frac{10}{t}\right) \frac{d t}{t}\right) \geqslant 2.64056 \quad \text { и } \quad F(2)=e^{\gamma},
$$

имеем

$$
20 e^{-\gamma}\left(f(5)-\frac{1}{2} \int_{3}^{10} F\left(5-\frac{10}{t}\right) \frac{d t}{t}\right)-4 \cdot 0.493 \cdot e^{-\gamma} \cdot F(2) \geqslant(1.32-1) 2 \approx 0.64
$$

поэтому справедливо неравенство

$$
\sum_{n_{1}}^{\prime} S\left(n_{1}\right) \geqslant \Lambda \frac{x}{\log ^{2} x}(0.64+O(\alpha)) \sum_{n_{1}}^{\prime} \frac{g\left(n_{1}\right)}{\varphi\left(n_{1}\right)}
$$

и можно считать, что доказано неравенство (2).

Перейдем к непосредственному доказательству теоремы. Предположим, что по некоторой последовательности $x_{l}$

$$
\lim _{x_{l} \rightarrow \infty} \frac{1}{\pi\left(x_{l}\right)}\left|\sum_{p \leqslant x_{l}} f(p+1)\right|=1 .
$$

Имеем

$$
\frac{1}{\pi\left(x_{l}\right)}\left|\sum_{p \leqslant x_{l}} f(p+1)\right|=\frac{1}{\pi\left(x_{l}\right)} \sum_{p \leqslant x_{l}} f(p+1) e^{-i \varphi\left(x_{l}\right)}
$$

где

$$
\varphi\left(x_{l}\right)=\arg \sum_{p \leqslant x_{l}} f(p+1) .
$$

Выделим из $x_{l}$ подпоследовательность, для которой $\varphi\left(x_{l}\right) \rightarrow \varphi_{0}$. Тогда

$$
\frac{1}{\pi\left(x_{l}\right)} \sum_{p \leqslant x_{l}} e^{i \varphi_{0}} f(p+1) \rightarrow 1 .
$$


Введем функцию

$$
f_{1}(n)= \begin{cases}f(n), & \text { если }(n, 2)=1, \\ f\left(2^{r}\right) e^{-i \varphi_{0}}, & \text { если } n=2^{r} .\end{cases}
$$

Тогда

$$
\frac{1}{\pi\left(x_{l}\right)} \sum_{p \leqslant x_{l}} f_{1}(p+1) \rightarrow 1 .
$$

Последнее равносильно соотношению

$$
\frac{1}{\pi\left(x_{l}\right)} \sum_{p \leqslant x_{l}}\left(1-\operatorname{Re} f_{1}(p+1)\right) \rightarrow 0
$$

Следовательно, с одной стороны мы имеем

$$
S_{1}=\frac{1}{\pi\left(x_{l}\right)} \sum_{\substack{n_{1} P_{2} \leqslant x_{l} \\ n_{1} P_{2}=p+1}}^{\prime}\left(1-\operatorname{Re} f_{1}\left(n_{1} P_{2}\right)\right) \rightarrow 0
$$

С другой стороны, мы доказали неравенство (2), по которому

$$
\sum_{n_{1}}^{\prime} S\left(n_{1}\right)=\sum_{\substack{n_{1} P_{2} \leqslant x_{l} \\ n_{1} P_{2}=p+1}}^{\prime} 1 \geqslant \delta \frac{x_{l}}{\log ^{2} x_{l}} \sum_{n_{1}}^{\prime} \frac{g\left(n_{1}\right)}{\varphi\left(n_{1}\right)}
$$

где $\delta>0$ - постоянная.

Теперь уточним дополнительные условия, накладьваемые на множество, которому принадлежит $n_{1}$. Пусть это множество таково, что $\left(1-\operatorname{Re} f_{1}\left(n_{1} P_{2}\right)\right)>\gamma>0$. Обозначим это множество $M=M\left(x^{\alpha}, \gamma\right)$. Тогда

$$
S_{1} \geqslant \frac{\gamma}{\pi\left(x_{l}\right)} \sum_{n_{1}}^{\prime} S\left(n_{1}\right) \geqslant \delta \frac{\gamma}{2 \pi\left(x_{l}\right)} \frac{x_{l}}{\log ^{2} x_{l}} \sum_{n_{1} \in M} \frac{g\left(n_{1}\right)}{\varphi\left(n_{1}\right)} .
$$

Покажем, что

$$
\sum_{n_{1} \in M} \frac{g\left(n_{1}\right)}{\varphi\left(n_{1}\right)} \geqslant \gamma_{1} \log x, \quad \gamma_{1}>0
$$

тем самым получим противоречие с (6). Напомним, что $f\left(P_{2}\right)$ равно либо $\zeta$, либо $\zeta^{2}$. Если $e^{-i \varphi_{0}} \neq \zeta, \zeta^{2}$, то положим

$$
M=\left\{n_{1}: n_{1} \leqslant x^{\alpha}, f\left(n_{1}\right)=1, n_{1} \text {-четное }\right\} .
$$

Если $e^{-i \varphi_{0}}=\zeta$, то в описании множества $M$ условие $f\left(n_{1}\right)=1$ заменим на $f\left(n_{1}\right)=\zeta^{2}$. При $e^{-i \varphi_{0}}=\zeta^{2}$ заменим условие $f\left(n_{1}\right)=1$ на $f\left(n_{1}\right)=\zeta$. При таком выборе множества $M$, как нетрудно видеть, неравенство $\left(1-\operatorname{Re} f_{1}\left(n_{1} P_{2}\right)\right)>\gamma$ вьполняется. Докажем неравенство (7) в случае $f\left(n_{1}\right)=1$. При $\sigma \leqslant \alpha$ имеем

$$
\sum_{n_{1} \in M} \frac{g\left(n_{1}\right)}{\varphi\left(n_{1}\right)} \geqslant \frac{1}{3} \sum_{n_{1} \mid P\left(x^{\sigma}\right)}^{\prime \prime} g\left(n_{1}\right) \frac{1+f\left(n_{1}\right)+f^{2}\left(n_{1}\right)}{\varphi\left(n_{1}\right)}-\sum_{n_{1} \mid P\left(x^{\sigma}\right), n_{1}>x^{\alpha}} \frac{g\left(n_{1}\right)}{\varphi\left(n_{1}\right)} ;
$$


$\sum^{\prime \prime}$ означает, что $n_{1}-$ четное число.

Пусть $G(n)$ - мультипликативная функция, которая равна либо 1 , либо $f\left(n_{1}\right)$, либо $f^{2}\left(n_{1}\right)$. Тогда

$$
S^{*}=\sum_{n_{1} \mid P\left(x^{\sigma}\right)}^{\prime \prime} \frac{g\left(n_{1}\right) G\left(n_{1}\right)}{\varphi\left(n_{1}\right)}=\prod_{2<p \leqslant x^{\sigma}}\left(1+\sum_{r=1}^{\infty} \frac{g\left(p^{r}\right) G\left(p^{r}\right)}{\varphi\left(p^{r}\right)}\right) \sum_{r_{1}=1}^{\infty} \frac{G\left(2^{r}\right)}{\varphi\left(2^{r}\right)} .
$$

Пусть $G(p)=1$. Тогда, так как $g\left(p^{r}\right)=g(p)$, имеем

$$
S^{*}=\prod_{p \leqslant x^{\sigma}}\left(1+\frac{g(p)}{p-1} \frac{p}{p-1}\right) \geqslant 3 \prod_{2<p \leqslant x^{\sigma}}\left(1+\frac{1}{p-2}\right) \geqslant \delta_{1} \log x^{\sigma}, \quad \delta_{1}>0 .
$$

Если $G(p)=\zeta$ или $\zeta^{2}$, то, так как $\operatorname{Re} \zeta=\operatorname{Re} \zeta^{2}=-1 / 2$, имеем

$$
\left|S^{*}\right| \ll \exp \left(-\frac{1}{2} \sum_{p \leqslant x^{\sigma}} \frac{1}{p}\right) \ll\left(\log x^{\sigma}\right)^{-1 / 2}
$$

Вычитаемое в правой части неравенства (8) не превосходит

$$
\begin{aligned}
\frac{1}{\alpha \log x} \sum_{n_{1} \mid P\left(x^{\sigma}\right)} \frac{g\left(n_{1}\right)}{\varphi\left(n_{1}\right)} \log n_{1} & \leqslant \frac{1}{\alpha \log x} \sum_{r, p \leqslant x^{\sigma}} \frac{\log p^{r}}{\varphi\left(p^{r}\right)} \sum_{n_{1} \mid P\left(x^{\sigma}\right)} \frac{g\left(n_{1}\right)}{\varphi\left(n_{1}\right)} \leqslant \frac{2 \sigma}{\alpha} \sum_{n_{1}} \frac{g\left(n_{1}\right)}{\varphi\left(n_{1}\right)} \\
& \leqslant \frac{2 \sigma}{\alpha} \cdot 3 \cdot \prod_{\substack{p \leqslant x^{\sigma} \\
p \neq 2}}\left(1+\frac{1}{p-2}\right) \ll \sigma^{2} \log x .
\end{aligned}
$$

Таким образом, подставляя найденные оценки в (8), получим

$$
\sum_{n_{1} \in M} \frac{g\left(n_{1}\right)}{\varphi\left(n_{1}\right)} \geqslant \gamma_{1} \log x, \quad \gamma_{1}=\delta_{1} \sigma+O\left(\sigma^{2}\right)>0
$$

при малых $\sigma$.

Следовательно, в случае $f\left(n_{1}\right)=1$ неравенство (7) и тем самым теорема доказаны.

Если $f\left(n_{1}\right)=\zeta$ или $f\left(n_{1}\right)=\zeta^{2}$, заменим $\left(1+f\left(n_{1}\right)+f^{2}\left(n_{1}\right)\right)$ в $(8)$ на $\left(1+\zeta^{2} f\left(n_{1}\right)+\right.$ $\left.\left(\zeta^{2} f\left(n_{1}\right)\right)^{2}\right)$ или $\left(1+\zeta f\left(n_{1}\right)+\left(\zeta f\left(n_{1}\right)\right)^{2}\right)$ соответственно и проведем аналогичные рассуждения.

Автор благодарит профессора Н.М. Тимофеева за полезные обсуждения.

\section{СПИСОК ЦИТИРОВАННОЙ ЛИТЕРАТУРЫ}

[1] Meyer J. Thèse d'Etat. Reims: Université de Reims, 1982.

[2] Hildebrand A. Additive and multiplicative functions on shifted primes // Proc. London Math. Soc. (3). 1989. V. 59. P. 209-232.

[3] Тимофеев Н. М. Мультипликативные функции на множестве сдвинутых простых // Изв. AH CCCP. Сер. матем. 1991. T. 55. C. 1238-1256.

[4] Elliott P. D. T. A. The multiplicative group of rationals generated by the shifted primes. I // J. Reine Angew. Math. 1995. V. 463. P. 169-216.

[5] Тимофеев Н. М. Неравенства Харди-Рамануджана и Халоса для сдвинутых простых чисел // Матем. заметки. 1996. Т. 57. № 5. С. 747-764.

[6] Halberstam H., Richert H.-E. Sieve Methods. London: Acad. Press, 1974. 